Available online at GSC Online Press Directory

GSC Biological and Pharmaceutical Sciences

e-ISSN: 2581-3250, CODEN (USA): GBPSC2

Journal homepage: https://www.gsconlinepress.com/journals/gscbps

(CASE REPORT)

\title{
Hemoglobin hammersmith associated with hemolytic anemia in a 3-year-old Colombian girl
}

\author{
González Figueredo Oscar Eduardo 1, ${ }^{*}$, Medellin Reina Sara Valentina ${ }^{2}$ and Guerrero Aislant Maria Camila ${ }^{2}$ \\ ${ }^{1}$ Pediatric hematologist \& oncologist. Fundación Santa Fé de Bogotá. Hospital central de la policía nacional de \\ Colombia. Bogotá DC, Colombia. \\ ${ }^{2}$ Universidad de los Andes. Bogotá DC, Colombia. \\ Publication history: Received on 13 April 2020; revised on 21 April 2020; accepted on 23 April 2020
}

Article DOI: https://doi.org/10.30574/gscbps.2020.11.1.0102

\begin{abstract}
Hemoglobin Hammersmith is a rare unstable variant of hemoglobin with decreased oxygen affinity. We report the case of a 3-year-old Colombian girl that initially consulted due to a presumptive diagnosis of iron deficiency anemia. During the evaluation we noticed typical features of chronic hemolytic anemia such as hepatosplenomegaly, jaundice and indirect hyperbilirubinemia; with the presence of target cells and intraerythrocytic inclusions with appearance of tactoids in the peripheral blood smear. The diagnosis of hemoglobin Hammersmith was confirmed through DNA sequencing revealing a pathogenic heterozygous variant in the beta globin chain gene due to a de novo mutation.
\end{abstract}

Keywords: Hemolytic anemia; Unstable hemoglobin; Hemoglobin Hammersmith; DNA analysis.

\section{Introduction}

Currently more than 1500 variants of hemoglobin have been described and approximately 150 are classified as unstable. Hemoglobin Hammersmith is known for being the second most common unstable hemoglobinopathy. These variants of hemoglobin are characterized by molecular changes that produce easy deoxygenation with a lower solubility of hemoglobin, causing secondary precipitation and formation of intra-erythrocyte inclusions. Thus, leading to erythrocyte damage and chronic hemolysis of varying severity. This instability is the product of a structural alteration in the globin chains as a result of point mutations, specifically in the beta-globin chain.[1]

We present the case of a 3-year-old female patient with a chronic non-immune hemolytic anemia associated with an electrophoretically silent hemoglobin, secondary to hemoglobin Hammersmith. Having knowledge of this pathology, can lead physicians to suspect its presence in cases of chronic non-immune hemolytic anemia with an apparently normal hemoglobin electrophoresis. By suspecting this hemoglobin subtype a timely molecular study can be made and subsequently obtain better diagnostic precision.

\section{Case presentation}

A 3-year-old Colombian girl is referred for outpatient pediatric hematology consultation with a presumptive diagnosis of iron deficiency anemia. She has a history of jaundice that was first noticed at 5 months of age with blood workup positive for macrocytic anemia, reticulocytosis, indirect hyperbilirubinemia and a negative direct Coombs test. Bone marrow biopsy revealed a normocellular bone marrow with erythroid relationship inversion due to marked erythroid hyperplasia and megaloblastic changes in the red line, without the presence of malignant cells. The patient was born by normal vaginal delivery at term, as the first child of non-consanguineous parents with a birth weight of 2,550 grams. Family history includes hypothyroidism in the mother that started during pregnancy, mild preeclampsia and long-

\footnotetext{
* Corresponding author: González Figueredo Oscar Eduardo. E-mail adress: oscarfigueredo777@hotmail.com
} 
standing jaundice. Also, her maternal grandmother presented diabetes mellitus type 2 and hypothyroidism. Further past medical history is negative apart from iron supplementation during 45 days at 5 months of age. Parents report that the patient presents occasional abdominal pain predominantly at night and deny episodes of drowsiness, fatigue, or respiratory distress in the patient.

At physical examination, the patient was hemodynamically stable with adequate oxygenation, scleral jaundice and a palpable spleen $5 \mathrm{~cm}$ below the left costal flank. The rest of the exam was within normal limits. Given the patient's history and findings, we began the study for hemolytic anemia. Peripheral blood smear revealed dianocytes and suggested the presence of tactoids which indicated a likely diagnosis of hemoglobin $\mathrm{C}$ disease. Direct and fractional Coombs were reported negative. Hemoglobin ( $\mathrm{Hb}$ ) electrophoresis in alkaline medium reported: $\mathrm{Hb} \mathrm{A}: 77.5 \%, \mathrm{Hb}$ A2: 3.2\%, Fetal Hb: 19.3\% whereas in acid medium: Hb A: 69.6\%, Hb A1 plus fetal Hb: 30.4\%. Erythrocyte osmotic fragility was normal. Glucose-6-phosphate- dehydrogenase and pyruvate kinase levels were normal. Flow cytometry for paroxysmal nocturnal hemoglobinuria was negative. Abdominal ultrasound reported splenomegaly of 122 millimeters, as the only remarkable finding without biliary lithiasis. Echocardiogram was negative for pulmonary hypertension.

After initial evaluation the patient persisted with mild anemia and predominantly indirect hyperbilirubinemia, without dyspnea or fatigue. All the while maintaining a normal appetite, with her growth being appropriate for her sex and age; at 50th weight percentile and 15th height percentile. Furthermore, red blood cell transfusion demand is low with requirement of one annual transfusion when the anemia was exacerbated by viral infections. Figures 1 to 6 show the evolution of different hematological parameters related to chronic hemolytic anemia of the patient.

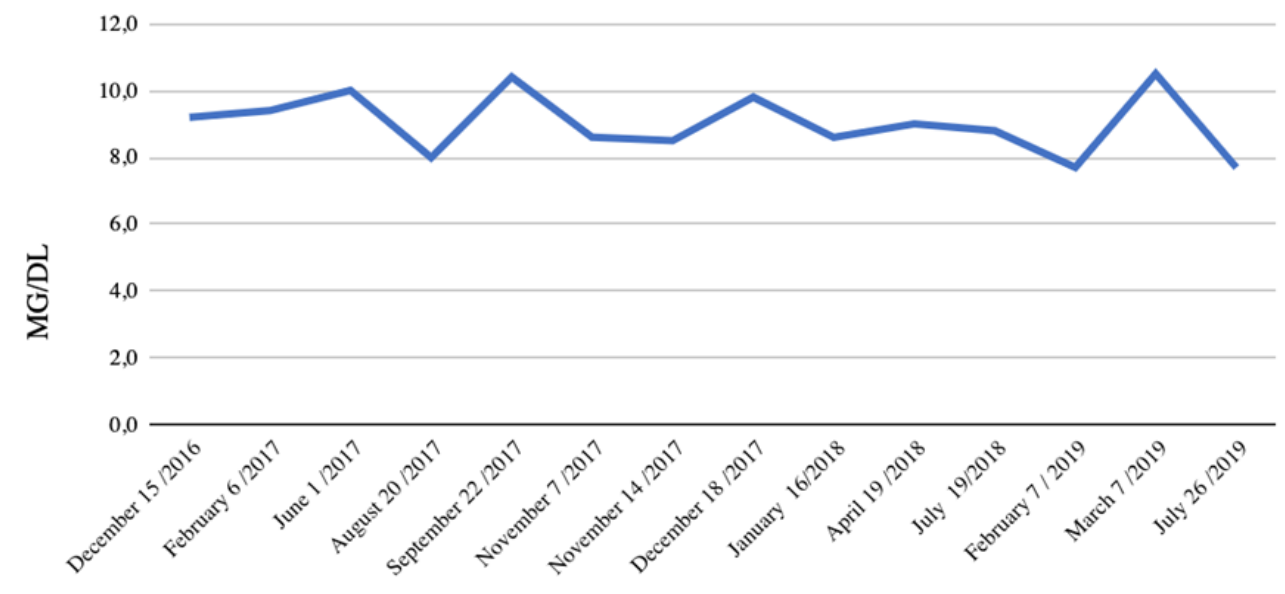

Figure 1 Hemoglobin levels

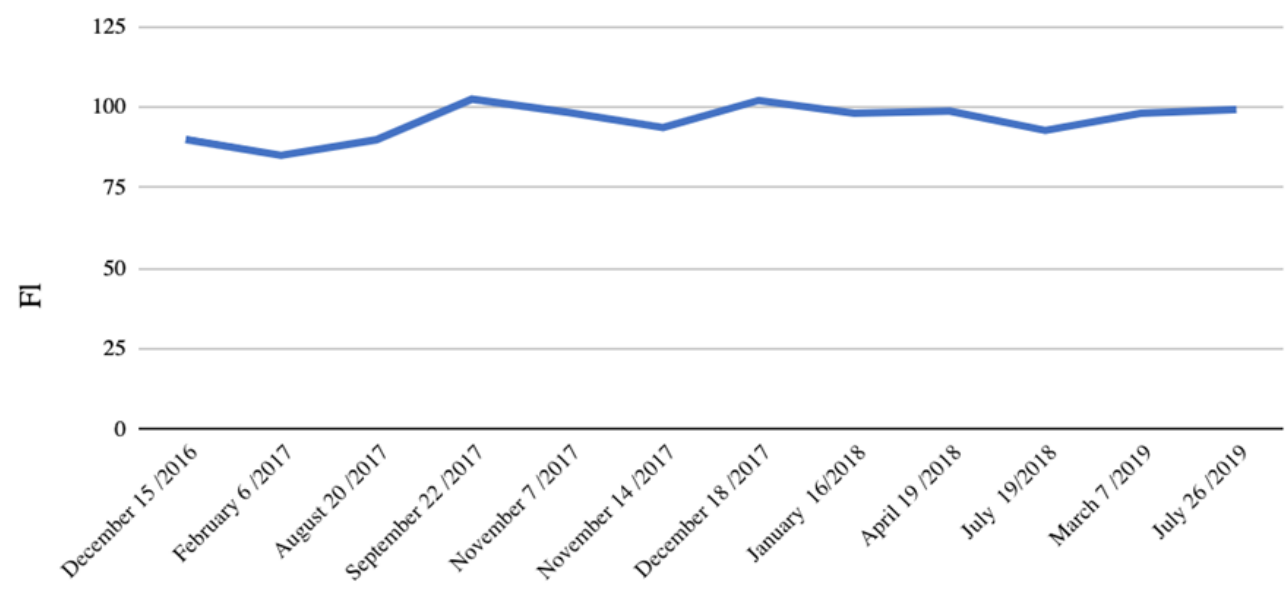

Figure 2 Mean corpuscular volume 
30

20

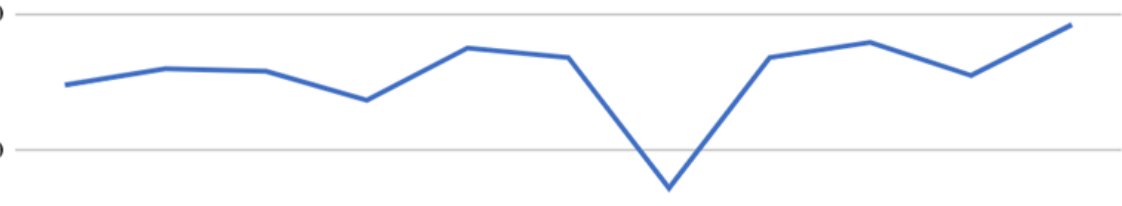

๖ 10

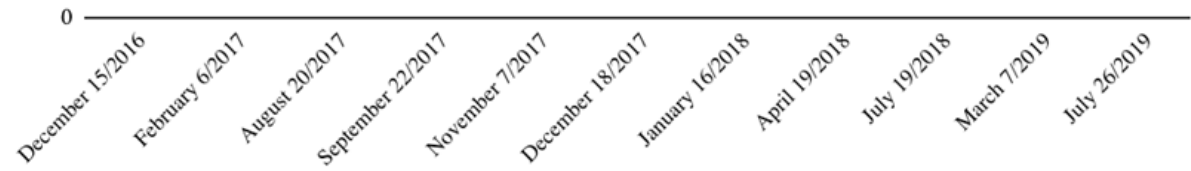

Figure 3 Red blood cell distribution width

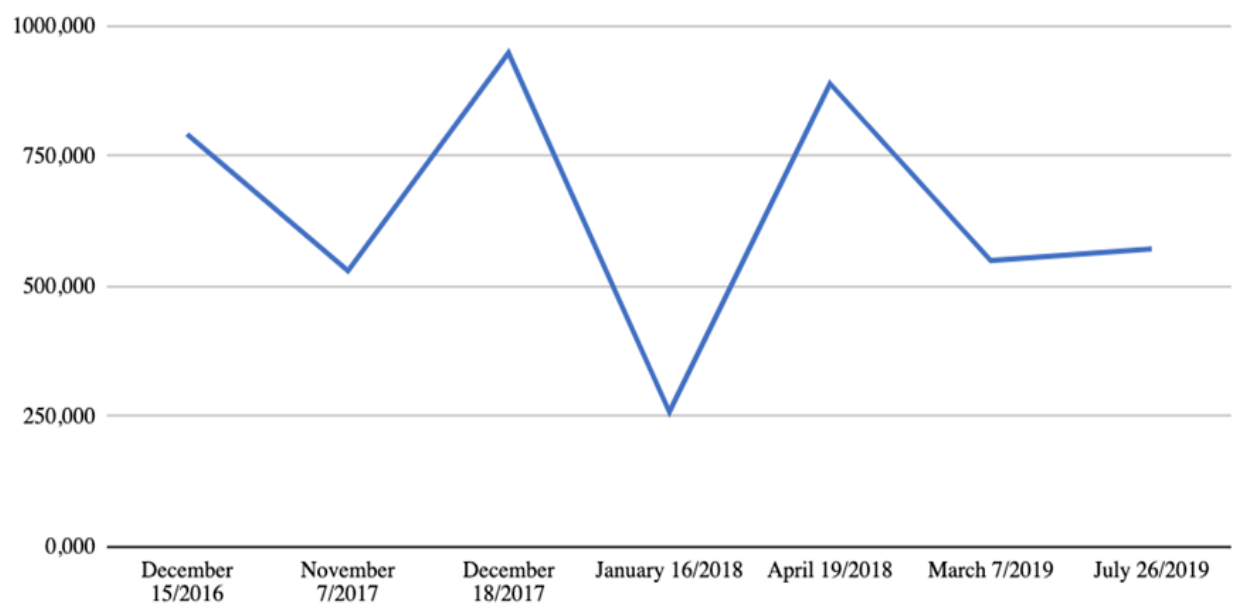

Figure 4 Reticulocyte count $/ \mu \mathrm{L}$.

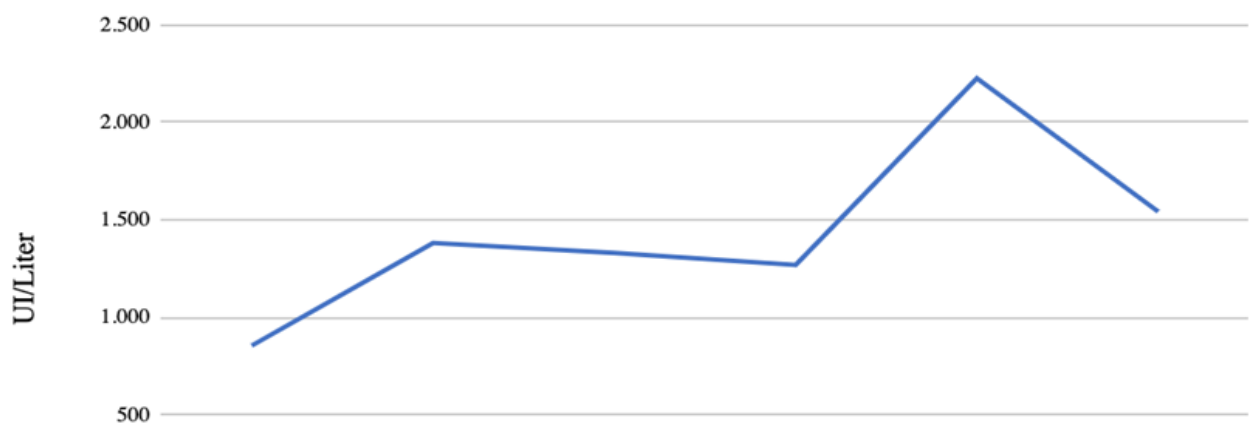

Februay 6/2017 August 20/2017 November 7/2017 December 18/2017 January 16/2018 $\quad$ April 19/2018

Figure 5 Lactate dehydrogenase levels 


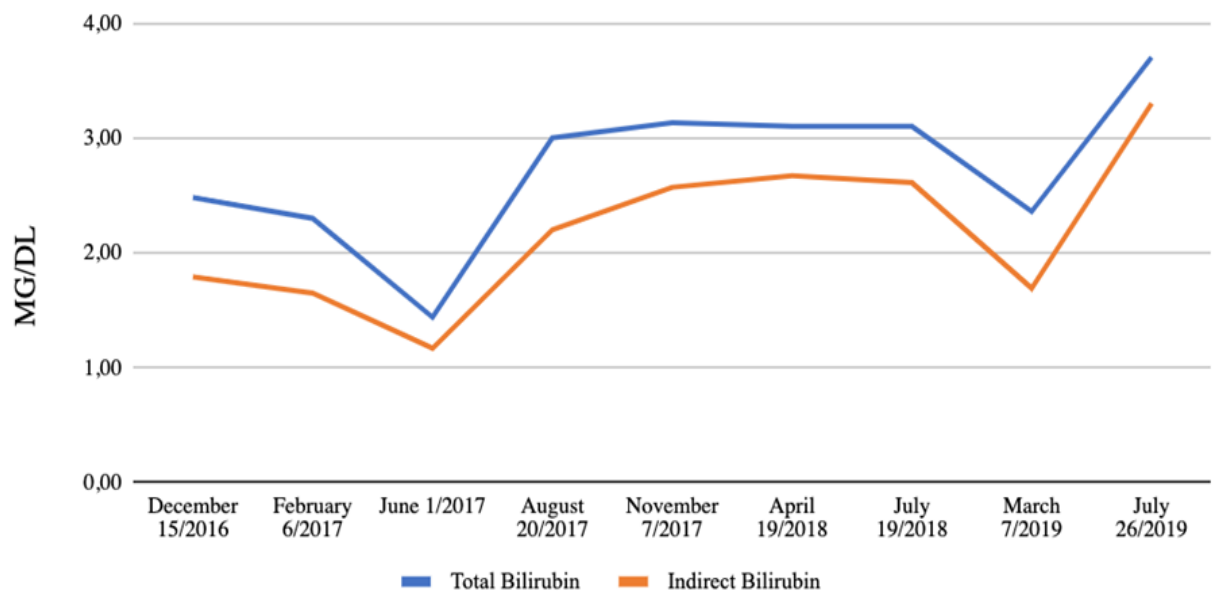

Figure 6 Bilirubin levels

Given the patient's clinical features, laboratory findings and a silent hemoglobin electrophoresis that revealed a significantly high hemoglobin $\mathrm{F}$ fraction, we suspected an unstable hemoglobinopathy. Exome genetic study was requested in order to analyze the genes of the globin chains. It reported a pathogenic heterozygous variant in the beta globin chain gene (c.128T $>C$ ) due to a de novo mutation. This variant is known as hemoglobin Hammersmith, confirming that the patient's non-immune hemolytic anemia was due to an unstable hemoglobinopathy.

\section{Discussion}

Hemoglobin Hammersmith is a variant of hemoglobin in which a mutation occurs in the beta chain of the molecule. There is a substitution of phenylalanine for a serine at the level of codon 42 ( $\beta 42$ (CD1) Phe $\rightarrow$ Ser) that induces a denaturation and precipitation of the hemoglobin molecule, giving rise to the formation of hemichromes. ${ }^{[2]}$ The end result is an unstable hemoglobin molecule that has a decreased affinity for oxygen and it leads to membrane damage with early erythrocyte destruction.[3]

The first case was described in 1964 by Grimes et al. [4], while studying a female patient with "idiopathic anemia with Heinz bodies" at Hammersmith Hospital in London, England (Royal Postgraduate Medical School, Hammersmith Hospital). Since then, approximately 19 cases of this disease have been reported in the literature.[5] Cases have been reported in different ethnic groups and most have been females with only two patients being male. While most described cases of hemoglobin Hammersmith are reported as spontaneous mutations, this condition behaves in an autosomal dominant pattern. The carrier has a $50 \%$ chance of passing the diseased allele to an offspring.

The molecular abnormality of hemoglobin Hammersmith produces a partial change in the functional properties of the structure ${ }^{[6]}$, which is therefore not detected with routine hemoglobin electrophoresis as it is not reflected by abnormal banding [7]. However, some studies have reported abnormal levels of $\mathrm{HbF}$ and $\mathrm{HbA2}{ }^{[8,9,10]}$, which can be seen in this case with our patient presenting high levels of $\mathrm{HbF}$.

The preferred method for the diagnosis of hemoglobin Hammersmith is by mutation detection with DNA analysis [11]. The HBB, HBA1 and HBA2 gene sequences are analyzed by gene-specific polymerase chain reaction (PCR) and the HBB: c. $128 \mathrm{~T}>\mathrm{C}$ (TTT $>$ TCT, Phe $\rightarrow$ Ser) mutation at codon 42 of the beta globin gene is the confirmatory outcome to diagnose this condition. Early prenatal diagnosis can be made, as presented by one reported case through beta-globin gene sequencing from DNA extracted from chorionic villi sampling ${ }^{[8]}$.

The clinical presentation of the unstable hemoglobinopathies may vary from asymptomatic to severe hemolysis resulting in anemia, splenomegaly, jaundice and pigmenturia. The complications are given by exacerbation of the hemolytic anemia secondary to oxidative stress caused by infections or medications, and gallbladder lithiasis which is frequent in chronic hemolytic processes ${ }^{[1]}$. Supportive treatment is given and splenectomy may be considered for the management of recurrent severe hemolytic anemia. 


\section{Conclusion}

Unstable hemoglobins are rare variants that should be suspected in patients with chronic nonimmune hemolytic anemia without a clear etiology and with silent hemoglobin electrophoresis; nonetheless high levels of fetal hemoglobin should not exclude the diagnosis. Hemoglobin Hammersmith is second unstable hemoglobin in frequency, highlighting the importance of knowing the pathology. Supportive treatment is given and splenectomy may be considered for the management of severe cases with high transfusion demand. Knowing this pathology allows us to suspect it in cases of chronic nonimmune hemolytic anemia; favoring accurate and timely diagnostic confirmation by DNA analysis.

\section{Compliance with ethical standards}

\section{Acknowledgments}

The authors want to thank Juliana Pérez Pinzón from Universidad de Los Andes, Bogotá DC, Colombia for critical revision of the manuscript.

\section{Disclosure of conflict of interest}

The authors have no conflicts of interest to declare.

\section{Author's contribution}

Each author contributed to the writing of this manuscript, as well as the analysis of the data and the literature reviewed in the discussion of this patient case.

\section{Statement of informed consent}

The patient's family provided written informed consent for the publication of this case report

\section{References}

[1] Eberle SJ, Noguera N, Calvo K, Ojeda M, Bragós I, Pratti A and Bonduela M. (2009). Anemia hemolítica grave causada por hemoglobina Hammersmith. Arch Argent Pediatr, 107(4), 347.

[2] Cunningham TA, Baker F, Kobrinskyl NL, Cepreganova B, Baysal E, Wilson JB and Huiwnan THJ. (1992). The unstable $\mathrm{Hb}$ Hammersmith or $\alpha 2 \beta 242$ (CD1) Phe $\rightarrow$ Ser observed in an Indian child; identification by HPLC and by sequence analysis of amplified DNA. Hemoglobin, 16(1-2), 19-25.

[3] Lang, SA, Chang, PC, Laxdal VA and Huisman TH. (1994). Haemoglobin Hammersmith precludes monitoring with conventional pulse oximetry. Canadian journal of anaesthesia, 41(10), 965-968.

[4] Grimes AJ, Meisler A and Dacie JV. (1964). Congenital Heinz-body anaemia: further evidence on the cause of Heinz-body production in red cells. British journal of haematology, 10(3), 281-290.

[5] Park S, Kang HJ, Cho SI, Kim SY, Seong MW and Park SS. (2012). A Case Report of a Male Patient With Hb Hammersmith [ $\beta 42$ (CD1) Phe $\rightarrow$ Ser, TT T> TCT]. Hemoglobin, 36(2), 161-165.

[6] Wajcman H, Leroux A and Labie, D. (1973). Functional properties of hemoglobin Hammersmith. Biochimie, 55(2), 119-126.

[7] Hung CC, Chien SC, Su YN, Chern JP, Lin KH and Lin WL. (2006). Denaturing high-performance liquid chromatography: an efficient screening approach in the genetic diagnosis of Hemoglobin Hammersmith. Biomedical Engineering: Applications, Basis and Communications, 18(06), 343-347.

[8] Li R, Wang T, Xie XM and Li DZ. (2014). Case report: prenatal diagnosis of Hb Hammersmith [ $\beta 42$ (CD1) Phe $\rightarrow$ Ser; HBB: c. 128T> C] in a family with an adult male patient. Hemoglobin, 38(2), 142-145.

[9] Dianzani I, Ramus S, Cotton RG and Camaschella, C. (1991). A spontaneous mutation causing unstable Hb Hammersmith: detection of the $\beta 42$ TTT $\rightarrow$ TCT change by CCM and direct sequencing. British journal of haematology, 79(1), 127-129.

[10] Sonati MF, Kimura EM, Abreu CF, Oliveira DM, Pinheiro VRP and Costa FF. (2006). Hemoglobin Hammersmith [ $\beta 42$ (CD1) Phe $\rightarrow$ Ser] in a Brazilian girl with congenital Heinz body hemolytic anemia. Pediatric blood \& cancer, $47(6), 855-856$. 
[11] Akiyama M, Murayama S, Yokoi K, Yanagisawa T, Hattori Y, Yamashiro Y and Fujisawa K. (2006). Hemoglobin Hammersmith [ $\beta 42$ (CD1) Phe $\rightarrow$ Ser] causing severe hemolytic anemia in a Japanese girl. Pediatric blood \& cancer, 47(6), 839-841.

\section{How to cite this article}

González Figueredo OE, Medellin Reina SV and Guerrero Aislant MC. (2020). Hemoglobin Hammersmith associated with hemolytic anemia in a 3-year-old Colombian girl. GSC Biological and Pharmaceutical Sciences, 11(12), 185-190. 